\title{
Minimizing Cytosol Dilution in Whole-Cell Patch-Clamp Experiments
}

\author{
Samsoon Inayat, Lawrence H. Pinto, and John B. Troy
}

\begin{abstract}
During a conventional whole-cell patch clamp experiment, diffusible cytosolic ions or molecules absent in the pipette solution can become diluted by a factor of one million or more, leading to diminished current or fluorescent signals. Existing methods to prevent or limit cytosol diffusion include reducing the diameter of the pipette's orifice, adding cytosolic extract or physiological entities to the pipette solution, and use of the perforated patch clamp configuration. The first method introduces measurement error in recorded signals from increased series resistance and the latter two are cumbersome to perform. In addition, most perforated patch configurations, prevent investigators from using test compounds in the pipette solution. We present a method to overcome these limitations by minimizing cytosol dilution using a novel pipette holder. After obtaining cellattached configuration conventionally with a large reservoir of pipette solution, we displace most of the pipette solution with mineral oil before going whole-cell thus minimizing cytosol dilution. To accomplish this requires a suction line and two Ag/AgCl electrodes inside the pipette. Testing our novel pipette holder with Chinese Hamster Ovarian cells, we demonstrate cytosol dilution factors between 76 and 234 . For large cells with somas greater than $\mathbf{4 0} \mu \mathrm{m}$, cytosol dilution factors of 10 or less are achievable.
\end{abstract}

Index Terms-Electrophysiology, patch-clamp, whole-cell, cytosol, rundown, pipette holder

\section{INTRODUCTION}

$\mathrm{T}$ he whole-cell patch-clamp configuration is a variant of the patch-clamp technique [1] [2], used to study membrane embedded proteins and their role in cellular physiology and

Manuscript received September 16, 2012. This work was supported in part by the National Science Foundation under Grant DBI-0551852. Samsoon Inayat was also supported by a graduate assistantship from the McCormick School of Engineering and Applied Sciences, Northwestern University and the Palmer Roberts Endowment.

Samsoon Inayat was with the Biomedical Engineering Department, Northwestern University, Evanston, IL 60208, USA. He is now with the Neurobiology Department, Northwestern University, Evanston, IL 60208, USA (email: sam@northwestern.edu) and Mechanical \& Industrial Engineering Department, University of Illinois, Chicago, IL 60607, USA (inayat@uic.edu)

Lawrence H. Pinto is with the Neurobiology Department, Northwestern University, Evanston, IL 60208, USA (email: larry-pinto@ northwestern.edu)

John B. Troy is with the Biomedical Engineering Department, Northwestern University, Evanston, IL 60208, USA (phone: 847-491-3822; fax: 847-4914928; email: j-troy@ northwestern.edu).

Copyright (c) 2013 IEEE. Personal use of this material is permitted. However, permission to use this material for any other purposes must be obtained from the IEEE by sending an email to pubs-permissions@ ieee.org.

pathophysiology. In this configuration, the physical system is a whole-cell surrounded by an extracellular medium and an essential part of the probing instrument is a glass micropipette containing an electrolyte solution and an electrochemical transducer, an $\mathrm{Ag} / \mathrm{AgCl}$ wire (Fig. 1). The $\mathrm{Ag} / \mathrm{AgCl}$ wire connects to electronic circuitry, which either clamps a cell's membrane voltage with respect to a ground $\mathrm{Ag} / \mathrm{AgCl}$ electrode placed in the extracellular medium or clamps the current passing through the membrane. In a typical whole-cell experiment, the stimuli provided to and the responses recorded from a cell are one or a combination of electrical, chemical, electromagnetic, thermal, or mechanical signals. Stimulusresponse datasets so acquired are used to build physiological models for cellular biology.

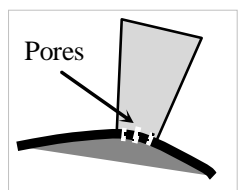

Perforated patch configuration

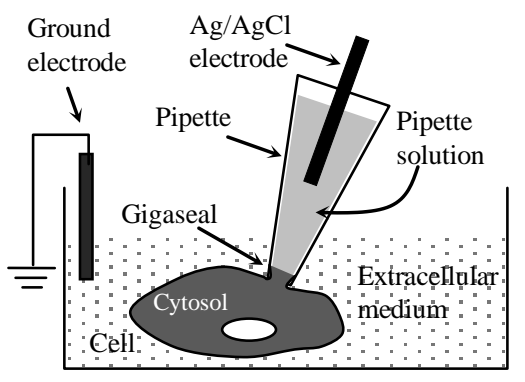

Fig. 1. Conventional whole-cell and perforated (inset) patch-clamp configurations. The dilution factor of an ion or molecule in the cytosol not present in the pipette solution is usually one million or more in conventional whole-cell configuration. The perforated patch-clamp configuration is achieved by using chemicals to create pores in the membrane allowing electrical recordings but preventing the passage of large molecules into the pipette solution and hence their dilution in the cytosol.

The first step towards obtaining whole-cell configuration is the achievement of cell-attached configuration, in which the cell is attached to the pipette through a mechanochemical seal between the pipette tip and the cell membrane. The seal is called a gigaseal owing to its electrical resistance in $\mathrm{G} \Omega \mathrm{s}$, and is obtained by touching the pipette tip against the cell membrane and applying slight suction inside the pipette while observing pipette resistance increase from $M \Omega$ s to G $\Omega$ s. After obtaining gigaseal, whole-cell configuration is achieved by puncturing the piece of cell membrane entrapped in the pipette tip, either by applying more suction through the pipette, an electrical pulse (zap) through the $\mathrm{Ag} / \mathrm{AgCl}$ electrode, or by impalement with a sharp metal wire [3]. This disruption of the membrane reduces the series resistance between the $\mathrm{Ag} / \mathrm{AgCl}$ and the ground electrodes, because of the ion channels in the plasma membrane, and increases capacitance, because the small capacitance due to the entrapped piece of cell membrane is 
replaced now by the larger capacitance of the whole-cell's plasma membrane. Hence, whole-cell configuration may be detected by an increase in capacitive transients apparent in the current waveform recorded in response to a voltage clamp pulse. Obviously, in whole-cell configuration, the cytosol and pipette solution are free to mix. How much and how quickly the cytoplasmic concentration of a particle (ion or molecule) changes depends on the initial concentrations of the particle in the cytosol and pipette solutions, the divergence of the product of the diffusion coefficient and the concentration gradient (Fick's second law of diffusion), and the geometry of the diffusion path, notably the area of the pipette orifice. The dependence on divergence should take account of the rate of generation and consumption of the particle.

In conventional whole-cell configuration, the volume of the pipette solution is usually more than one million times that of the cytosol. The pipette solution typically contains 5-15 different types of ions and molecules depending upon the type of investigation [4]. The cytosol however contains a far larger number (at least in the hundreds) of different types of diffusible ions and molecules [5, 6], all involved in different cellular mechanisms. Some cytosolic compounds are in such low concentration that they number close to a thousand molecules [7]. In many whole-cell patch-clamp experiments, the dilution of the constituents of the cytosol is ignored as a first approximation, assuming that it has a minimal effect on the stimulus-response relationship under consideration. This assumption is often invalid and, in some experiments, cytosol dilution causes a rundown of the recorded signals themselves (e.g. channel currents or intracellular fluorescence) [8-13].

To prevent or limit rundown of the signals through diffusion from the cytosol, experimenters have used one or a combination of the following methods:

\section{Reducing pipette orifice diameter}

The pipette tip presents the smallest geometric area in the electrohydraulic pathway; so reducing the size of its opening increases resistance to ionic and molecular diffusion, delaying rundown. But, the reduction in ionic flow also increases the electrical series resistance, which introduces voltage-clamp errors and limits the bandwidth of recorded signals $[14,15]$.

2. Adding cytosolic extract or functional constituents to the pipette solution

Adding cytosolic extract to the pipette solution can theoretically eliminate concentration gradients between the cytosol and the pipette, but this is very difficult to do. Adding functional constituents such as ATP, cAMP, leupeptin or phosphatase inhibitors to the pipette solution can prevent dephosphorylation and proteolysis, reducing signal rundown, but perhaps at the expense of altering intracellular signaling systems[12, 16-18].

\section{Perforated patch-clamp configuration}

As the name suggests, perforations (pores in Fig.1 inset) are created in the membrane patch (rather than a puncture) while in cell attached configuration by using ionophores including ATP, polyene antiobiotics (nystatin and amphotericin B), gramicidin, and $\beta$-escin $[12,16]$. The size of the pores varies for the different ionophores, thus leading to different rundown delaying characteristics. Series resistance in all perforated patch configurations is higher than with conventional whole-cell and, except the use of $\beta$ escin, all other ionophores prohibit the addition of test compounds to the pipette solution. It is also difficult to get gigaseals with ionophores present in the pipette solution and, in some instances, perforated patch recordings move spontaneously into whole-cell configuration [19].

These remedies to limit cytosol diffusion have helped experimenters since the mid 1980's in numerous different types of study. However, there remains considerable room for improvement. In the ideal situation, we would like to be able to probe the cell using the whole-cell patch-clamp technique in a controlled manner to investigate its physiology; i.e., with low series resistance and the ability to control the composition and volume of the pipette solution. We introduce a novel motorized pipette holder and whole-cell configuration, which we believe is one step closer to the ideal than the conventional method. With the novel pipette holder, we achieve a whole-cell configuration with low series resistance and substantially reduced cytosol dilution while allowing the addition of test compounds to the pipette solution.

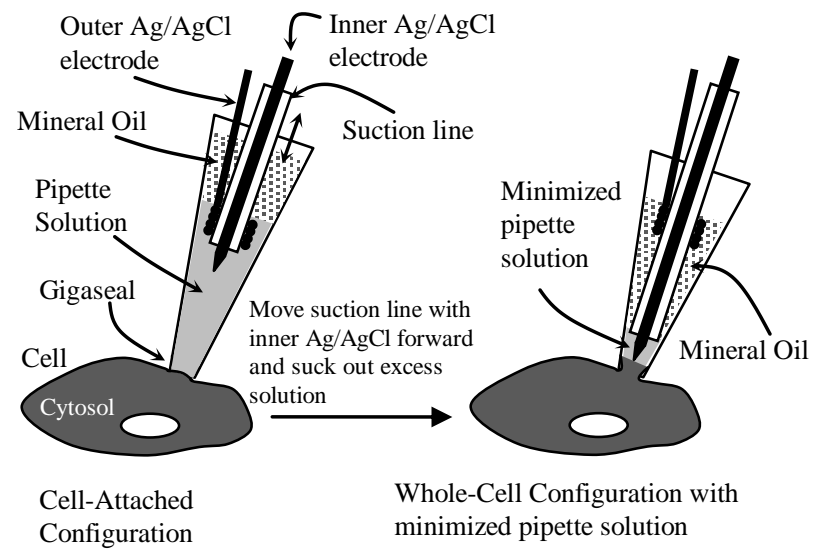

Fig. 2. System to minimize cytosol dilution in the whole-cell configuration. The pipette is filled with pipette solution backed with mineral oil. The pipette contains a linearly movable assembly of an inner $\mathrm{Ag} / \mathrm{AgCl}$ electrode (with a conical tip) and a suction line. The outer $\mathrm{Ag} / \mathrm{AgCl}$ electrode is fixed. First, a cell-attached configuration is obtained in the conventional way with a large reservoir of pipette solution while the assembly of the inner $\mathrm{Ag} / \mathrm{AgCl}$ and suction line sits far back in the pipette (Left). Here the outer $\mathrm{Ag} / \mathrm{AgCl}$ electrode is connected with the amplifier to detect formation of the gigaseal. After obtaining gigaseal, the inner $\mathrm{Ag} / \mathrm{AgCl}$-suction line assembly is moved forward close to the pipette tip and excess pipette solution is removed from the pipette through the suction line, and replaced by the mineral oil (Right). The inner $\mathrm{Ag} / \mathrm{AgCl}$ electrode is then connected to the amplifier and a zap or zap combined with suction is applied to disrupt the cell membrane, moving to whole-cell configuration with minimal pipette solution.

In order to achieve a low resistance whole-cell configuration while minimizing cytosol dilution, we reduce the volume of the pipette solution by using a mechanohydraulic method implemented with a novel pipette holder. In the novel pipette holder, there are two $\mathrm{Ag} / \mathrm{AgCl}$ electrodes and a suction line, inside the pipette (Fig. 2). The two $\mathrm{Ag} / \mathrm{AgCl}$ electrodes can be connected separately to the amplifier. The outer $\mathrm{Ag} / \mathrm{AgCl}$ electrode is fixed in position. The inner $\mathrm{Ag} / \mathrm{AgCl}$ electrode (with a conical tip) is combined with the suction line in an 


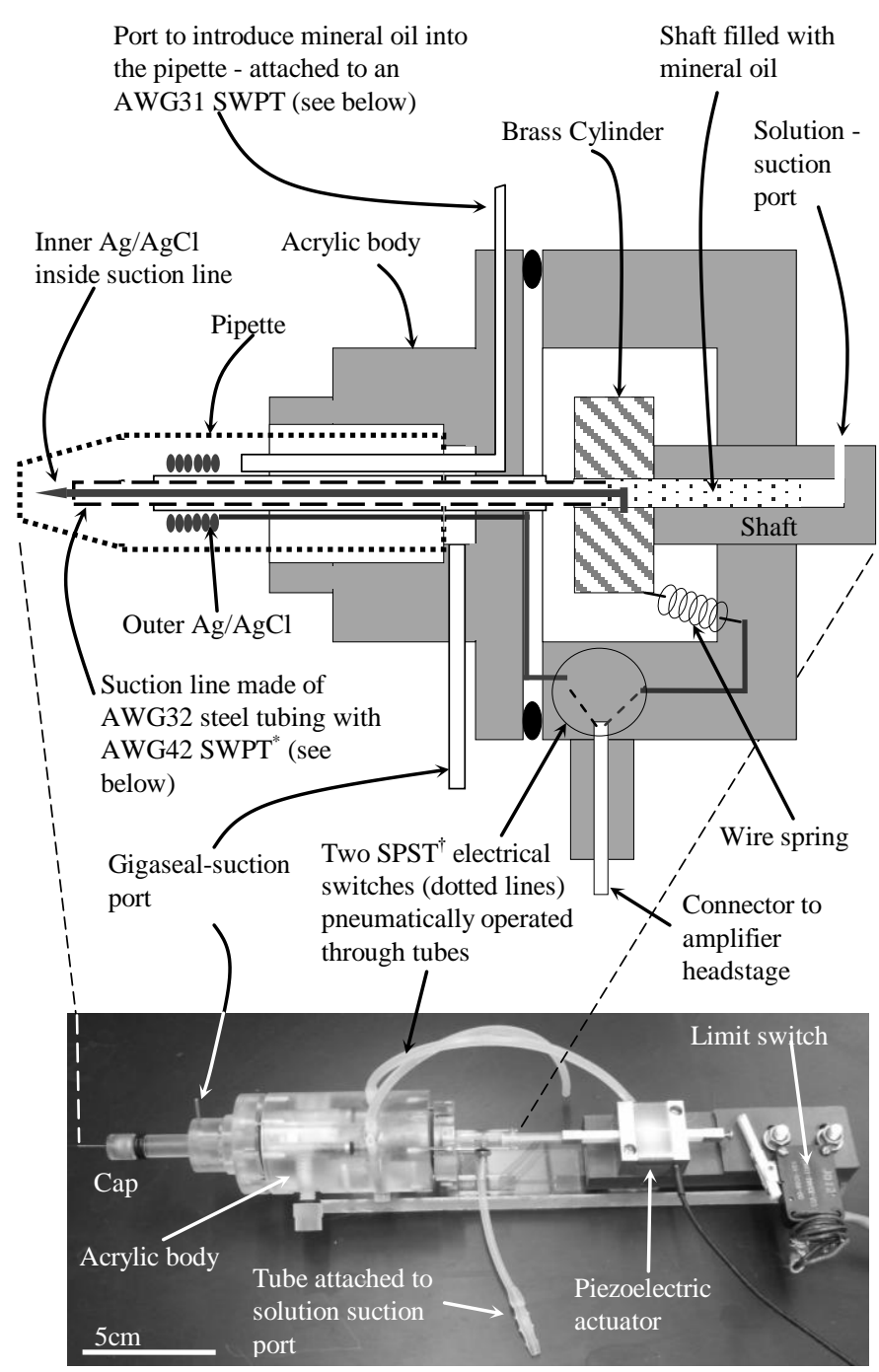

$\uparrow$ SPST - Single-Pole-Single-Throw

* SWPT - Single-Walled-Polyimide Tubing

Concentric AWG 30 and 28 SWPTs acting as guide for the linear movement of AWG32 steel tube


Fig. 3. Schematic (top) and photograph (middle) of the motorized pipette holder with two $\mathrm{Ag} / \mathrm{AgCl}$ electrodes and a suction line inside the pipette (bottom). The two $\mathrm{Ag} / \mathrm{AgCl}$ electrodes can be connected individually to the amplifier using two single-pole-single-throw (SPST) electrical switches operated pneumatically. There are two suction ports, one for obtaining a gigaseal and the other for removal of excess pipette solution and producing whole-cell configuration.

integrated assembly that moves linearly within the pipette. Using the outer $\mathrm{Ag} / \mathrm{AgCl}$ we first obtain cell-attached configuration in the conventional way with a large reservoir of pipette solution (Fig. 2-left). Then we move the integrated inner $\mathrm{Ag} / \mathrm{AgCl}$-suction line assembly close to the pipette tip (Fig. 2-right) and remove excess pipette solution through the suction line while replacing it with mineral oil initially backfilled into the pipette. At this point, the outer $\mathrm{Ag} / \mathrm{AgCl}$ is surrounded by insulating mineral oil and the inner $\mathrm{Ag} / \mathrm{AgCl}$ electrode is inside the small volume of pipette solution near the tip. The inner $\mathrm{Ag} / \mathrm{AgCl}$ is then connected to the amplifier and a zap or a zap combined with suction is applied to disrupt the cell membrane and obtain whole-cell configuration. Testing our method with Chinese Hamster Ovarian (CHO) cells we demonstrate a cytosol dilution factor (dilution factor of an ion or molecule not present in the pipette solution) of 234 or less as compared to $\sim 1,000,000$ or more for the conventional whole-cell configuration. Cytosol dilution factors of 10 or less are achievable with cells having bodies of $40 \mu \mathrm{m}$ diameter or greater (e.g., dorsal root ganglion cells) with our device and method.

\section{MATERIALS AND METHODS}

\section{A. The Novel Motorized Pipette Holder}

Figure 3 shows schematic drawings and a photograph of the motorized pipette holder that attaches to the headstage $(\mathrm{CV}$ 201A) of an Axopatch 200A amplifier (Molecular Devices, Inc., CA, USA). The housing of the holder was machined from an acrylic rod (Small Parts Inc., Amazon, WA, USA) and mounted on an acrylic plate. The housing with the acrylic plate mounts on the amplifier headstage vertically as opposed to the horizontal mounting of a conventional pipette holder. This $90^{\circ}$ rotation of the headstage allowed the pipette holder to incorporate a large piezoelectric linear actuator (N310 NEXACT $^{\circledR}$, Physik Instrumente, GmbH, Germany), which, through a shaft, moves the integrated assembly of an inner $\mathrm{Ag} / \mathrm{AgCl}$ electrode and a suction line inside the pipette (see Fig. 2). Around the integrated assembly, there is an immovable outer $\mathrm{Ag} / \mathrm{AgCl}$ electrode. The two $\mathrm{Ag} / \mathrm{AgCl}$ electrodes can be separately connected to the amplifier via two pneumatically operated single-pole-single-throw (SPST) electrical switches incorporated in the holder housing. To supply suction for gigaseal formation and to later both extract pipette solution and obtain the whole-cell configuration, there are two separate suction lines. One incorporated in the holder housing and the other inside the movable shaft. The text below describes construction of each of the above mentioned components. 
Linear Shaft, Suction Line, and Inner Ag/AgCl Electrode: The hollow shaft is made from an acrylic rod (Small Parts) and incorporates a suction line to remove most of the pipette solution. At one end it connects to the linear actuator and at the other to a piece of AWG32 steel tubing (Small Parts) through a brass cylinder. At the end of the steel tube, glued opposite to the brass cylinder, is a piece of concentric AWG42 single walled polyimide tubing - SWPT (Small Parts). This serves to reduce the diameter of the suction line where it reaches into the pipette tip close to the cell. A $50 \mu \mathrm{m} \mathrm{Ag}$ wire (Alfa Aesar, Ward Hill, MA, USA) sits inside the suction line. One end of the $\mathrm{Ag}$ wire is connected to the brass cylinder and the other end is conical and coated with $\mathrm{AgCl}$. The conical end serves as the inner $\mathrm{Ag} / \mathrm{AgCl}$ electrode and protrudes $\sim 0.5 \mathrm{~mm}$ from the AWG42 SWPT. A wire spring connects the brass cylinder to the amplifier through a pneumatically operated electrical switch (construction explained below). Assembly of the AWG32 steel tubing, the AWG42 SWPT and the $50 \mu \mathrm{m}$ $\mathrm{Ag}$ wire with the conical $\mathrm{AgCl}$ tip was done manually with the aid of an upright optical microscope.

Outer Ag/AgCl Electrode and Polyimide Tubing Assembly: Figure 3 (bottom) shows the arrangement of polyimide tubes and outer $\mathrm{Ag} / \mathrm{AgCl}$ electrode inside the pipette. The inner most tube is a concentric combination of AWG 30 and 28 SWPT to enhance mechanical strength. The steel tube carrying the inner $\mathrm{Ag} / \mathrm{AgCl}$ electrode moves linearly inside the AWG 30 SWPT. In order to ensure that the AWG30 SWPT remains concentric with the pipette two AWG29 SWPTs are glued above and below the AWG28 SWPT (see front view). Inside the top AWG29 SWPT sits an AWG31 SWPT connected to a syringe filled with mineral oil. In the bottom AWG29 sits a $50 \mu \mathrm{m}$ $\mathrm{AgCl}$-coated $\mathrm{Ag}$ wire (Alfa Aesar), part of which is wound on the AWG 30 SWPT (see figure 3) to form the outer $\mathrm{Ag} / \mathrm{AgCl}$ electrode. The other end of the $\mathrm{Ag} / \mathrm{AgCl}$ electrode is connected to the amplifier through one of the pneumatic electrical switches. This assembly was also made manually under an upright optical microscope.

Two Pneumatically Operated Single-Pole-Single-Throw Electrical Switches: There are two vertically oriented U-type holes in the acrylic cylindrical body each partially filled with $0.1-0.2 \mathrm{ml}$ of liquid mercury $(\mathrm{Hg}$, available from GalliumSource, LLC through Amazon.com). One leg of a Utype hole is exposed to the atmosphere and the other is connected to a $1 \mathrm{ml}$ syringe used to control the height of the $\mathrm{Hg}$ column in the other leg. The leg exposed to atmosphere contains two electrical wires. One wire connects to the amplifier while the other connects to one of the $\mathrm{Ag} / \mathrm{AgCl}$ electrodes. When $0.1 \mathrm{ml}$ of air is injected through the syringe into one leg, the mercury rises in the other leg connecting the two wires electrically. The syringe is operated with a high precision Zaber TLA-28A linear actuator (Zaber Technologies, Inc., Vancouver, BC, Canada) which controls the rate of injection. The electrical resistance of both switches is less than $15 \Omega$.

\section{B. Pipette Loading Procedure}

The text below explains the procedure to load a pipette into the novel pipette holder, which takes 1-2 minutes, a little longer than loading a pipette in a conventional holder.

Homing the integrated suction line-inner $\mathrm{Ag} / \mathrm{AgCl}$ electrode assembly: The first step is to ensure that the integrated suction line with inner $\mathrm{Ag} / \mathrm{AgCl}$ electrode assembly is at its home (backmost) position, detected by a limit switch (Fig. 3). In this position the conical tip of the inner $\mathrm{Ag} / \mathrm{AgCl}$ sits safely inside the guide/protector tube, permitting pipette replacement without risk of damage.

Filling the pipette with solution: The pipette is filled with about $7 \mathrm{~mm}$ of solution, so that when fully loaded the solution barely touches the tip of the AWG31 SWPT (Fig. 3), while the outer $\mathrm{Ag} / \mathrm{AgCl}$ electrode is immersed completely.

Partial loading of the pipette onto the polyimide tubing assembly: The pipette filled with solution is then partially loaded onto the polyimide tubing assembly so that the air solution interface is about $3 \mathrm{~mm}$ or more from the tip of the guide tube (AWG30). This ensures that the solution does not come into contact with the polyimide tubing assembly at this time.

Flushing air from the protector tube: The suction line containing the inner $\mathrm{Ag} / \mathrm{AgCl}$ electrode is then advanced until it protrudes $\sim 1 \mathrm{~mm}$ from the protector tube, flushing any air trapped in the protector tube and preventing it entering the pipette solution.

Further loading of the pipette onto the polyimide tubing assembly.: The pipette is next loaded gradually further onto the polyimide tubing until the air solution interface is about $1 \mathrm{~mm}$ from the tip of the AWG31 SWPT. Care is taken to avoid bubbles entering the pipette solution from the protector tube.

Introduction of mineral oil: Mineral oil is injected through the AWG31 SWPT from a $3 \mathrm{ml}$ plastic syringe connected to the mineral oil port (Fig. 3). The oil forms an interface with the pipette solution flushing air away as it advances within the pipette. A length of $\sim 7 \mathrm{~mm}$ of mineral oil is filled in the pipette above the interface between oil and solution.

Full loading of the pipette: The pipette is now completely loaded in the pipette holder, the cap tightened to seal the interior of the pipette from air and the pipette tip then dipped into the bath solution.

\section{Silver (Ag) conical wires}

We built a semi-automatic setup to electrochemically etch $\mathrm{Ag}$ wires following the method of [20]. $50 \mu \mathrm{m}$ cylindrical Ag wire (Alfa Aesar) was dipped $3 \mathrm{~mm}$ into $30 \%$ ammonium hydroxide $\left(\mathrm{NH}_{4} \mathrm{OH}\right)$ solution containing a steel ground electrode. $1.7 \mathrm{~V}$ was applied to the $\mathrm{Ag}$ wire above ground using the analog output of an NIDAQ-USB 6259 (National Instruments, TX, USA) data acquisition card (DAQ) as the wire was moved upwards at a rate of $5 \mu \mathrm{m} / \mathrm{s}$ using a linear drive. The current through the wire was measured using an analog input of the DAQ card and a sharp drop in the current indicated the completion of etching. The tip of the resulting conical silver wire was coated with $\mathrm{AgCl}$ by dipping it $300 \mu \mathrm{m}$ into physiological saline while applying $1.5 \mathrm{~V}$ above ground for 30 secs. 


\section{Glass Pipette Fabrication}

Pipettes were fabricated as described before [3]. Briefly, 5 $\mathrm{cm}$ pipettes with a cone length of 4-5 $\mathrm{mm}$, a pipette tip opening of $1-2 \mu \mathrm{m}$ and a cone angle between $10^{\circ}$ and $15^{\circ}$ (close to the tip opening) were pulled from Corning 8161 patch glass tubes (10 cm length, $1.5 \mathrm{~mm}$ OD and $1.1 \mathrm{~mm}$ ID) with a Sutter P-87 Glass Puller (Sutter Instruments, Novato, CA, USA).

\section{E. Cell Culture}

Chinese Hamster Ovarian (CHO-K1) cells were cultured as described before [3]. Briefly, they were cultured on $25 \mathrm{~cm}^{2}$ flasks in F-12 Kaighn's modification medium (Hyclone, Logan, UT, USA) containing $10 \%$ fetal bovine serum (Sigma-Aldrich, MO, USA) and $1 \%$ penicillin-streptomycin (Invitrogen, Carlsbad, CA, USA). For patch-clamping they were cultured on glass coverslips in a $35 \mathrm{~mm}$ petri dish.

\section{F. Whole-Cell Patch-Clamping}

Whole-cell patch-clamping was done using the same setup as described before [3]. Briefly, whole-cell configuration was achieved for $\mathrm{CHO}-\mathrm{K} 1$ cells at room temperature $\left(22-25^{\circ} \mathrm{C}\right)$ and with a holding potential of $0 \mathrm{mV}$. An Axopatch 200A (Molecular Devices, CA, USA) amplifier equipped with a Digidata 1440A (Molecular Devices) and Clampex 10.2 (Molecular Devices) data acquisition software were used. Current signals were low-pass filtered with a $2 \mathrm{kHz}$ setting on the Axopatch 200A and digitized at $10 \mathrm{kHz}$.

The orientation of the headstage on the setup was rotated by $90^{\circ}$ to fit our motorized pipette holder. The bath solution contained in $\mathrm{mM}$; N-methyl-D-Glucamine (NMDG) (135), HEPES (25), $\mathrm{CaCl}_{2}$ (5), and glucose (10), with $\mathrm{pH}$ adjusted to 7.4 with methanesulfonic acid. The pipette solution contained in mM; N-methyl-D-Glucamine (NMDG) (135), HEPES (25), and EGTA (5), with pH adjusted to 7.2 with methanesulfonic acid. The pipette resistance ranged between 4-10 M $\Omega$.

\section{G. Fluorescence and Imaging}

$100 \mu \mathrm{M}$ fluorescein-5-(and-6)-sulfonic acid, trisodium salt (Invitrogen) was added to the pipette solution to permit fluorescent confirmation of whole-cell configuration. Fluorescein was excited at $480 \mathrm{~nm}$ through a 20x objective lens (Olympus ULWD CDPlan 20 PL) with NA 0. Imaging was done with a 1.3 Megapixels Moticam 1000 camera (Motic, Xiamen, China) with Motic Image Plus 2.0 image acquisition software. The camera was mounted on the eye piece of the patch-clamp setup microscope Olympus IMT-2. To capture Clampex membrane test data and images from Motic synchronously, a custom matlab (The Mathworks, Inc) script was written to capture screen images at a sampling interval of $3 \mathrm{~s}$.

\section{H. Modeling and Simulation}

Modeling of cytosol diffusion from the cell into the pipette was done using COMSOL Multiphysics Software (COMSOL, Inc, Stockholm, Sweden) while also testing the idea of reducing pipette solution volume by plugging the pipette tip with the $\mathrm{Ag} / \mathrm{AgCl}$ wire. 2D finite element models of a cytosol-pipette solution system with a blunt-tip conical wire were made and the diffusion of particles was simulated with four diffusion coefficients and multiple wire positions. COMSOL's Transport of Diluted Species Interface was used to solve the timedependent diffusion equation

$$
\frac{\partial c}{\partial t}+\nabla \cdot\left(-D_{c} \nabla c\right)=0
$$

Where $\mathrm{c}$ is the concentration of the particle at a given time and space, $D_{c}$ is the diffusion coefficient of the particle in water. The diffusion was assumed to be isotropic. The initial concentrations of particle in the cytosol and pipette solution were $1 \mathrm{M}$ and $0 \mathrm{M}$ respectively. Geometrical details are given in Figure 4.
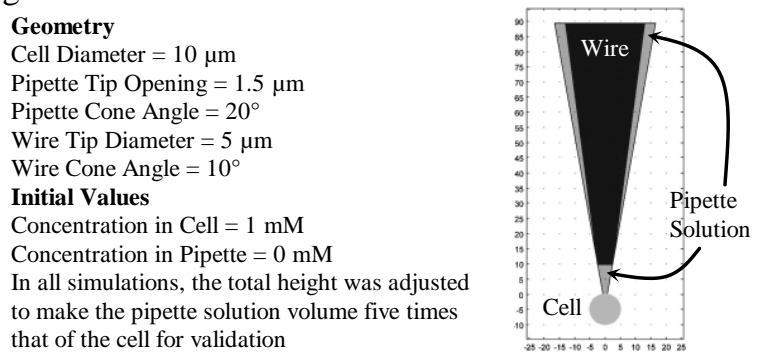

Fig. 4 Geometry of the cytosol-pipette solution system with a blunt-tip conical wire movable linearly in the pipette. The blunt-tip in its forward most position can in theory limit all but a tiny volume of pipette solution coming into contact with the cytosol in whole-cell configuration thus minimizing cytosol dilution.

The average element quality of mesh elements was greater than 0.95 in all simulations. The DIRECT time-dependent PARDISO solver was used. In order to validate simulations, the volume of the pipette solution was set to be 5 times that of the cell in all simulations by adjusting its height. This way, the steady state value of concentration of particle in the cell approaches $1 / 6$ of the initial concentration.

\section{RESUlTS AND DISCUSSION}

Our initial idea for preserving cytosol integrity in patch clamp experiments was to prevent cytosol diffusion into the pipette solution by filling the pipette orifice with an $\mathrm{Ag} / \mathrm{AgCl}$ wire. In Inayat et al. (2010), we describe a new patch-clamp electrode employing what we call pushpen operation and believed that this system could be used for this purpose. In the pushpen operation a conical metal wire moves linearly inside the pipette and can protrude from its tip like a pushpen. We demonstrated the use of the pushpen operation to obtain whole-cell configuration from cell attached mode by impaling the cell membrane rather than by applying suction. We speculated that with the pushpen patch clamp system it might be possible to plug the hole in the cell membrane in whole-cell configuration with a conical metal tip and thus block the passage for cytosol diffusion. It turned out to be challenging to make a conical tip for the wire that creates a perfect seal, especially since pipette tips vary. Controlling the position of the wire accurately in the pipette tip is also a significant engineering challenge. Furthermore, from our experience with the pushpen operation (with $\mathrm{W}$ wire) we learnt that the membrane does not form a mechanochemical seal with the 
metal wire [3]. Hence, a natural progression of the idea was to try to minimize cytosol dilution (rather than prevent cytosol diffusion) by minimizing the volume of pipette solution contacting the cytosol in whole-cell configuration.

In order to develop a methodology to minimize cytosol dilution in whole-cell configuration, we first estimated what cell and pipette solution dimensions are required to achieve a certain degree of cytosol dilution, which we assess by defining a generic cytosol dilution factor (CDF) as

$$
\begin{aligned}
& \text { Cytosol Dilution Factor }(C D F)=\frac{C V+P S V}{C V} \\
& C V=\text { Cell Volume, PSV = Pipette Solution Volume }
\end{aligned}
$$

Here, we assume that the pipette solution is pure water and the cytosol volume is equivalent to half of the cell volume [7]. The smallest possible value of CDF is 1, indicating preserved cytosol in the whole-cell configuration and is only achievable if the membrane hole is filled completely by the $\mathrm{Ag} / \mathrm{AgCl}$ electrode. Hence, the nearer the value of CDF is to 1 , the less cytosol dilution. In a conventional whole-cell patch clamp experiment, $\mathrm{CDF}$ is greater than $1,000,000$ and sometimes as big as $3,000,000$.

A.
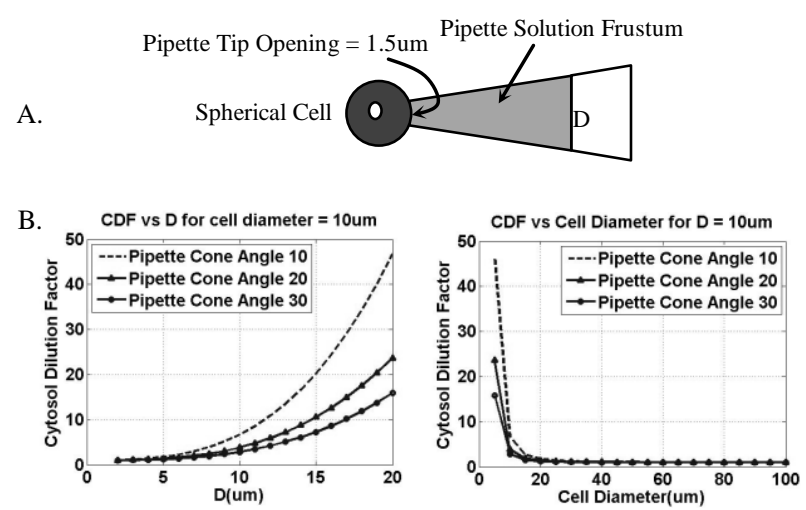

Fig. 5 Cytosol dilution factor (CDF) dependence on cell and pipette solution dimensions.

\section{A. CDF's dependence on cell and pipette solution dimensions}

To determine the pipette solution dimensions necessary to achieve a specific CDF for a particular cell size, we consider a spherical cell in whole-cell configuration with a pipette solution forming a frustum in the pipette tip (Figure 5A). Figure 5B shows plots of the CDF versus the pipette solution frustum base diameter (D) and cell diameter respectively for three pipette cone angles (typical for patch pipettes). For figure 5Bleft, the cell diameter is fixed at $10 \mu \mathrm{m}$, a typical value for an eukaryotic cell, and for figure 5B-right, D is fixed at $10 \mu \mathrm{m}$ (arbitrary value). In order to obtain a $\mathrm{CDF}<10$ for cell sizes greater than or equal to $10 \mu \mathrm{m}$, we note from figure $5 \mathrm{~B}$ that we require a value of $\mathrm{D}$ between 5 and $15 \mu \mathrm{m}$ for pipette cone angles varying between $10^{\circ}$ and $30^{\circ}$. We also require an $\mathrm{Ag} / \mathrm{AgCl}$ electrode in contact with or sitting inside the pipette solution frustum for electrical connection. The $\mathrm{Ag} / \mathrm{AgCl}$ electrode will therefore, have a conical tip with an apex diameter less than $\mathrm{D}$ and a cone angle smaller than that of the pipette interior.

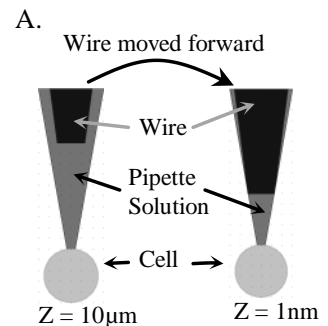

B.

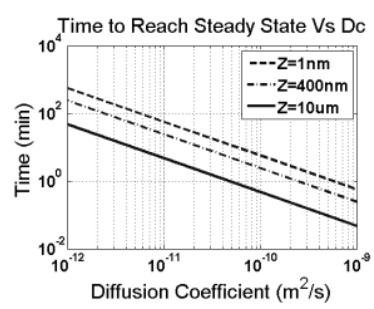

$\mathrm{Z}$ is vertical displacement of the wire from $\mathrm{Z}=0$

where it touches the pipette interior wall

Fig. 6 COMSOL modeling of cytosol diffusion. (A) Schematic of two wire positions in the pipette. $Z=10 \mu \mathrm{m}$, equivalent to a conventional whole-cell configuration and $\mathrm{Z}=1 \mathrm{~nm}$, almost complete fluid isolation in the tip (within practical limits of the finite element model). For $Z=1 \mathrm{~nm}$, there is a circumferential channel of $176 \mathrm{pm}$ between the wire edge and pipette's interior wall. (B) Time to reach steady state concentration versus diffusion coefficients (Dc) for three $\mathrm{Z}$ values. For modeling parameters, see Materials and Methods.

\section{B. Methodology to obtain a low CDF in whole-cell configuration}

A low CDF in whole-cell configuration cannot be achieved with a conventional pipette holder. One cannot restrict the pipette solution to as little as that shown in Fig. 5. Moreover, were this possible, one could not achieve whole-cell configuration, because formation of a gigaseal requires suction and the presence of the $\mathrm{Ag} / \mathrm{AgCl}$ wire close to the pipette tip impedes the transmission of suction applied inside the pipette to the cell membrane [3]. Hence, establishment of a low CDF requires first that one obtain the gigaseal with a large volume of pipette solution and then reduce this volume to establish a low CDF for the whole-cell configuration. To determine whether it would be preferable to reduce the quantity of pipette solution prior to or after going whole-cell we investigated numerically, using COMSOL (see $\mathrm{H}$ in Materials and Methods). We examined the dynamics of diffusion for biologically important molecules from the cytosol into the pipette while testing the approach of minimizing cytosol dilution by plugging the pipette tip with an $\mathrm{Ag} / \mathrm{AgCl}$ wire. Figure 6A-left mimics a cell and pipette solution in conventional whole-cell configuration. Figure 6B-solid line represents the time to reach a steady state cytosolic concentration for various diffusion coefficients (i.e. different ions or molecules). $\mathrm{Z}$ in the figure is the vertical displacement of the wire, measured from the point at which it fits tightly against the pipette's inner wall. As one might expect, the smaller is $\mathrm{Z}$, the slower the rundown of particles from the cytosol. Even when $\mathrm{Z}$ is just $1 \mathrm{~nm}$, molecules such as ATP having diffusion coefficients of the order of $10^{-10} \mathrm{~m}^{2} \mathrm{~s}^{-1}$ will equilibrate with the pipette solution within a few minutes of going whole-cell. When the $\mathrm{Ag} / \mathrm{AgCl}$ wire is far removed from the tip, as it is in a conventional patch pipette, equilibration occurs in seconds, seriously compromising the duration of an experiment. It is evident therefore that one must reduce the reservoir of pipette solution before going whole-cell. To validate the simulation results, we fabricated a patch clamp system with a blunt-tip inner $\mathrm{Ag} / \mathrm{AgCl}$ wire that, after obtaining the gigaseal, could be advanced linearly and moved to the most forward position in the pipette (i.e., so that the wire abuts the pipette's inner wall), in theory isolating the pipette solution in 
the tip from the bulk solution. In this position, a zap could be used to obtain whole-cell from the cell-attached configuration. Even though up to a 100 fold increase in pipette resistance was achievable when the $\mathrm{Ag} / \mathrm{AgCl}$ electrode was fully advanced, while testing the system in a saline bath, there was still a significant path for diffusion left. So, while one might prolong the duration of experiments by slowing cytosol dilution with this approach, one cannot maintain a low CDF.

\section{Design to obtain a low $C D F$}

The following text describes the structural and functional components of the mechanism to remove excess pipette solution to obtain a low CDF.

Suction Line and inner $\mathrm{Ag} / \mathrm{AgCl}$ Electrode: The obvious means to remove excess pipette solution is by suction, hence the need for a suction line inside the pipette. Based on the dimensions of the pipette solution to obtain a low CDF (Fig. 5), we require the suction line to be as small as possible and reach as close as possible to the pipette tip. This way most of the excess pipette solution can be removed. We used AWG42 single-walled polyimide tubing (SWPT) with an inner diameter (ID) of $60 \mu \mathrm{m}$ and an outer diameter (OD) of $86 \mu \mathrm{m}$ as the suction line that reached into the pipette tip. The AWG42 SWPT is attached to a piece of steel tubing (AWG32) at the back for mechanical strength (see Fig. 3 schematics). The AWG42 SWPT reaches into the pipette tip where the pipette ID is a little more than $86 \mu \mathrm{m}$. Within the suction line, there is a conical $\mathrm{Ag} / \mathrm{AgCl}$ electrode (etched from $50 \mu \mathrm{m}$ diameter $\mathrm{Ag}$ wire and coated with $\mathrm{AgCl}$ - see Materials and Methods) which contacts the reduced pipette solution.

Linear Motion of Suction Line: As discussed previously, the pipette tip must be unobstructed when making the gigaseal [3], hence the suction line and $\mathrm{Ag} / \mathrm{AgCl}$ electrode have to sit far back in the pipette and later advanced. This requirement necessitates the incorporation of a linear actuator into the pipette holder. We used a piezoelectric actuator (see Materials and Methods) having a minimum step size of $\sim 50 \mathrm{~nm}$. Due to the large dimensions of the actuator, we designed the pipette holder to attach to the patch-clamp amplifier headstage vertically. The linear actuator moves a hollow shaft inside the pipette holder housing. This shaft is attached to the suction line AWG32 steel tubing through a brass cylinder. The hollow hole in the shaft forms the port through which pipette solution is sucked. The $\mathrm{Ag} / \mathrm{AgCl}$ wire inside the suction line attaches to the brass cylinder which is connected to the amplifier by one of the pneumatically operated SPST switches (see Fig. 3). The hollow shaft is filled with mineral oil to electrically insulate the $\mathrm{Ag} / \mathrm{AgCl}$ wire from outside interference. Without the mineral oil, the pipette solution inside the suction line acts as an antenna and adds noise to signals carried by the $\mathrm{Ag} / \mathrm{AgCl}$ wire.

Protecting the conical tip of the inner $\mathrm{Ag} / \mathrm{AgCl}$ electrode: To avoid damaging the tip of the inner $\mathrm{Ag} / \mathrm{AgCl}$ electrode while loading or unloading pipettes, a "guide/protector tube" made from concentrically joined AWG30 and 28 SWPTs is used (Fig. 3). The suction line housing the electrode moves within the guide tube and the electrode tip will be undamaged when hidden there.

Concentricity of suction line with the pipette: To keep the suction line concentric with the pipette interior and add mechanical strength, two AWG29 SWPTs were attached to the guide tube, one on top and one below (see front view, Fig. 3) as "fillers". The combined width of the guide/filler tubes is 1.05 $\mathrm{mm}$ compared to the pipette's $1.1 \mathrm{~mm}$ ID. Without the AWG29 SWPTs the guide tube snags against the pipette's interior wall and removal of pipette solution from near the tip is no longer possible.

Use of mineral oil: Backfilling the pipette with mineral oil removes residual pipette solution between the tubes and the pipette interior wall, leading to isolation of the pipette solution at the tip. When changing pipettes, the mineral oil is loaded into the pipette after the pipette solution, through the AWG 31 SWPT (Fig 3), which sits inside the top filler tube (AWG29).

Detecting isolation of the tip solution: The detection of isolation of the pipette solution at the tip is achieved through optical imaging and electrical measurements. Optical imaging of the pipette tip alone can be misleading. Firstly, the microscope provides only the top view of the tilted pipette making it difficult to see any residual solution. Secondly, an aqueous channel of dimensions below optical resolution (e.g., less than $400 \mathrm{~nm}$ ) provides a large enough connection for significant diffusion to occur between the solution in the tip and any solution at the back of the pipette (Fig. 6B, dotted line for $Z=400 \mathrm{~nm}$ ). A criterion based on electrical measurements however detects a minute liquid connection. So we added a fixed outer $\mathrm{Ag} / \mathrm{AgCl}$ electrode (see Fig. 3) that sits far back in the pipette and used this to measure pipette resistance and capacitance while reducing the quantity of pipette solution at the tip. Pipette resistance increases and capacitance decreases when mineral oil surrounds the outer $\mathrm{Ag} / \mathrm{AgCl}$ electrode.

The outer $\mathrm{Ag} / \mathrm{AgCl}$ electrode serves the additional purpose of detecting the formation of the gigaseal. Because of the limited coat of $\mathrm{AgCl}$ on the inner $\mathrm{Ag} / \mathrm{AgCl}$ electrode, use of the outer $\mathrm{Ag} / \mathrm{AgCl}$ electrode during the measurement of pipette resistance and detection of the formation of the gigaseal helps preserve the $\mathrm{AgCl}$ on the inner $\mathrm{Ag} / \mathrm{AgCl}$ electrode for electrical recordings in whole-cell mode.

\section{Obtaining low CDF whole-cell configuration with Chinese Hamster Ovarian (CHO) cells}

How to obtain a low CDF whole-cell configuration with $\mathrm{CHO}$ cells is outlined next.

Loading pipette and employing the outer $\mathrm{Ag} / \mathrm{AgCl}$ electrode: The pipette is first loaded into the pipette holder using the procedure mentioned previously (see B in Materials and Methods). Then, the outer $\mathrm{Ag} / \mathrm{AgCl}$ electrode is connected to the amplifier, and, using the Clampex membrane test (with bath option), the pipette resistance measured and the offset on the amplifier adjusted to null the current for null voltage-clamp, removing any offset potential between the $\mathrm{Ag} / \mathrm{AgCl}$ electrode and the pipette solution.

Obtaining Cell-Attached Configuration: The pipette was then brought on top of the cell to be patched (Fig. 7A), and 
lowered to touch its tip with the cell membrane. Once this had been detected by an increase in pipette resistance, slight suction was applied through the solution-suction port (less than $1 \mathrm{ml}$ with a $60 \mathrm{ml}$ syringe). Following this, additional suction was applied through the gigaseal-suction port $(\sim 2 \mathrm{ml}$ with a $20 \mathrm{ml}$ syringe). While applying this suction the Clampex waveform was monitored to confirm formation of a gigaseal i.e. cellattached configuration (Fig. 7B). After obtaining the gigaseal, the gigaseal-suction port was opened to the atmosphere. Application of suction through both ports was a key step for increasing the success rate in obtaining gigaseals. When suction was applied only through the gigaseal-suction port, vacuum was lost when air is taken in from the solution-suction port.

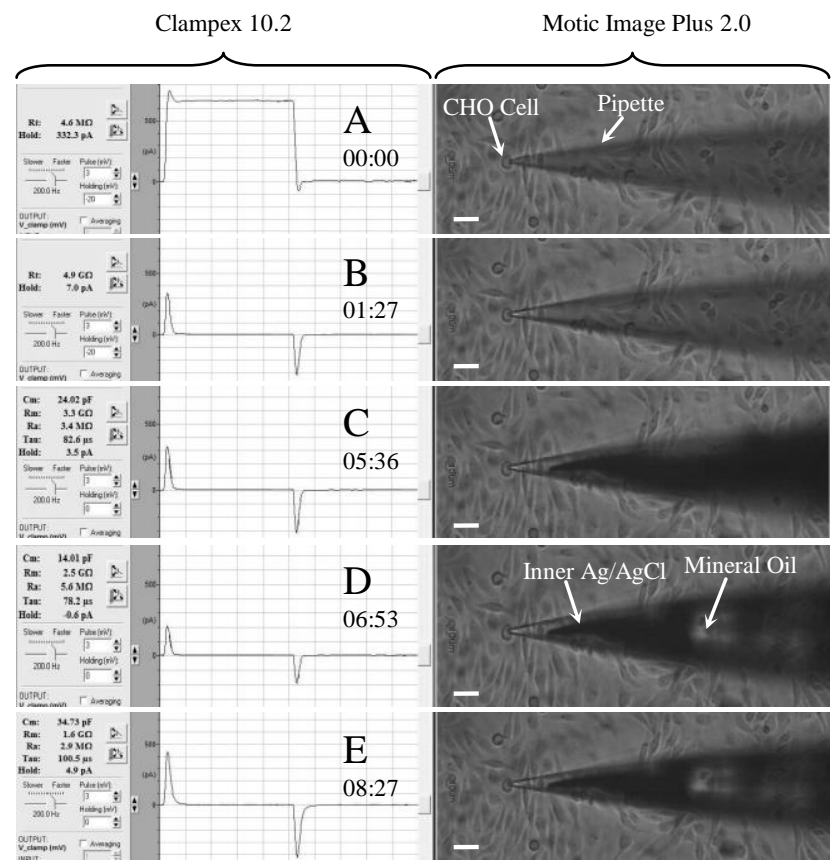

Fig. 7. Demonstration of obtaining a low CDF whole-cell configuration with CHO cells. A-E respectively show with time-stamps (mins:secs), pipette in bath, cell-attached configuration, movement of the suction line-inner $\mathrm{Ag} / \mathrm{AgCl}$ electrode assembly close to the pipette tip, suction and replacement of excess pipette solution with mineral oil, and whole-cell configuration. Scale bars $40 \mu \mathrm{m}$.

Obtaining the low CDF whole-cell configuration: After obtaining cell attached configuration (Fig. 7B), we paused the membrane test on Clampex and moved the suction line-inner $\mathrm{Ag} / \mathrm{AgCl}$ electrode assembly forward by activating the piezoelectric actuator. To prevent electrical or mechanical noise from breaking the gigaseal, the piezoelectric motor was driven with $5 \mathrm{~V}, 30 \mathrm{~Hz}$ stepping pulses, which moved its shaft at a speed of $\sim 1 \mathrm{~mm} \mathrm{~min}^{-1}$. At this speed it took $\sim 4$ mins for the tip of the inner $\mathrm{Ag} / \mathrm{AgCl}$ electrode to reach the pipette tip (Fig. 7C). During this motion $\pm 0.1 \mathrm{nA}$ variable noise was visible on the current waveform in Clampex, but this did not break the gigaseal. Motion was terminated when the conical tip was within $100 \mu \mathrm{m}$ of the tip (Fig. 7C). The membrane test in Clampex was then turned $\mathrm{ON}$ and about $30 \mathrm{ml}$ of constant suction applied with a $60 \mathrm{ml}$ syringe through the solutionsuction port. It took 1-2 mins to suck the solution from the pipette, replacing it with mineral oil. Once the oil surrounded the outer $\mathrm{Ag} / \mathrm{AgCl}$ electrode we observed a decrease in the capacitive transients (Fig. 7D). At this point, the mineral oil had still to isolate a small volume of solution at the pipette tip. When it did and the oil-pipette solution interface became visible in the microscope, the solution-suction port was exposed to air and closed (Fig. 7D). The outer $\mathrm{Ag} / \mathrm{AgCl}$ electrode was then disconnected from the amplifier by retracting the plunger of a syringe to remove $0.1 \mathrm{ml}$ of air from its mercury switch. Movement of the syringe was controlled by a Zaber linear actuator driven at a speed of $\sim 0.25 \mathrm{~mm} \mathrm{~s}^{-1}$. As the mercury moved in its column it introduced variable noise of up to $-0.2 \mathrm{nA}$ (offset below zero baseline) with low and high frequency transients. Faster movement of the actuator generated more noise, so a compromise was reached between noise reduction and the time needed to activate the switch. After disconnecting the outer $\mathrm{Ag} / \mathrm{AgCl}$ electrode, the offset for the inner $\mathrm{Ag} / \mathrm{AgCl}$ electrode was set on the amplifier. This offset was measured previously by connecting the inner $\mathrm{Ag} / \mathrm{AgCl}$ electrode with the amplifier. Another Zaber linear actuator was then advanced at $~ 0.25 \mathrm{~mm} \mathrm{~s}^{-1}$ to inject $0.1 \mathrm{ml}$ of air to activate the mercury switch used to connect this inner $\mathrm{Ag} / \mathrm{AgCl}$ electrode to the amplifier. The motion of mercury in this case introduced a variable noise of up to $+0.2 \mathrm{nA}$ (offset above zero baseline) with low and high frequency transients. When mercury contacted the two terminals of the switch, a large transient burst of high frequency switching noise was seen and acted as a zap to the membrane. In some cases, this took the recording into whole-cell mode. Sometimes it destroyed the gigaseal and sometimes it had no effect. When the gigaseal was lost, the experiment had to be abandoned. When the zap had no effect, a zap from the amplifier was applied while the membrane test was paused to obtain wholecell configuration. Suction through the solution-suction port was also found to help obtain whole-cell configuration while applying a zap. Whole-cell mode was signaled by increase in the capacitive transients (Fig. 7D-E) with the gigaseal intact. Using the Clampex capacitance measurement, we determined the capacitances of the pipette holder and mercury switches to be roughly 6 and $12 \mathrm{pF}$ respectively. With cells larger than 10 $\mu \mathrm{m}$ in size and in cell-attached configuration, the total capacitance measured in membrane test with either one of the $\mathrm{Ag} / \mathrm{AgCl}$ electrodes is less than $25 \mathrm{pF}$. In whole-cell mode it is above $30 \mathrm{pF}$.

Confirming attainment of whole-cell mode: To demonstrate that whole-cell mode had been achieved we added $100 \mu \mathrm{M}$ fluorescein-5-(and-6)-sulfonic acid trisodium salt (FSAT) to the pipette solution. Within 1-2 mins of obtaining whole-cell configuration, fluorescence was seen in the CHO cell (Fig. 8 A-C) consistent with the predictions of the COMSOL modeling study. The diffusion coefficient of FSAT is between $10^{-9}$ and $10^{-10} \mathrm{~m}^{2} \mathrm{~s}^{-1}$ based on its molecular weight 478.32 (close to that of ATP) and thus would diffuse at a rate similar to that of our simulation (Fig. 6 - right). The addition of FSAT also demonstrates the potential of the addition of other test compounds to the pipette solution for influencing cellular biochemistry.

Success rate of obtaining whole-cell configuration: We obtained cell-attached configuration with 18 cells, of which 8 were shifted successfully into whole-cell configuration with gigaseals greater than $1 \mathrm{G} \Omega$. For two other cells in "whole-cell 
configuration", one had a seal resistance in the range $0.5-1 \mathrm{G} \Omega$ and the other in the range 0.2-0.5 G $\Omega$. The other eight cells had their gigaseals destroyed by the zap. Thus after obtaining cell-attached configuration, our success rate of achieving whole-cell configuration is $44 \%$. The time to obtain whole-cell configuration after the achievement of gigaseal was $6.7 \pm 2.8$ minutes (mean \pm standard deviation, $\mathrm{n}=8$ ).

Maintenance of whole-cell mode: For 7 of the 8 cells wholecell recording was lost within 10 minutes. We attribute this rapid loss of recording to having not optimized the parameters used for the zap to obtain whole-cell configuration, which if optimized, should result in more stable whole-cell configurations. For the other cell, where the zap was better controlled, we were able to maintain the whole-cell recording for 33 minutes. This cell was fluorescent throughout (Fig. 8C) and maintained a stable gigaseal and series resistance (Fig. 8D) suggestive of its continued physiological activity. Electrophysiologists hypothesize that the increase in series resistance they often observe during whole-cell mode results either from loss of cellular integrity or from partial blockage when cytosol components migrate into the pipette tip. Our long term recording result is consistent with this hypothesis and we believe that minimizing cytosol dilution can improve the stability of series resistance.

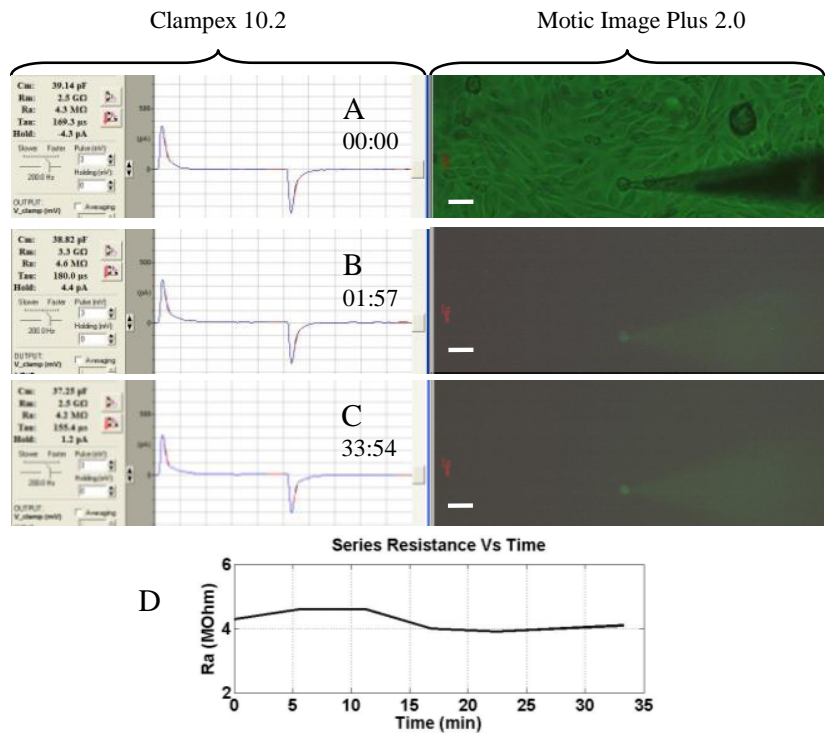

Fig. 8. Confirmation and stability of whole-cell configuration. A-C. Confirming whole-cell mode with fluorescin in the pipette solution. Within two minutes of obtaining whole-cell configuration (A), fluorescin from the pipette solution had diffused into the cell (B), whose fluorescence remained stable until the end of the recording (C). Scale bars $40 \mu \mathrm{m}$. D. Series resistance (Ra) versus time for the same cell is also stable.

Assessing the degree of cytosol dilution in whole-cell configuration: In order to estimate cytosol dilution factors, we estimated cell and pipette solution volumes from microscope images captured during whole-cell experiments. The cell volume is calculated assuming the $\mathrm{CHO}$ cell is spherical, a reasonable assumption. The pipette solution volume was determined by subtracting the inner $\mathrm{Ag} / \mathrm{AgCl}$ frustum volume from the pipette frustum volume. The table in Figure 9 shows data from 8 experiments. The mean value of CDF was 140 with a standard deviation of 60 , which compares very favorably when compared against a CDF of 1,000,000 with a conventional patch-clamp system in whole-cell mode. Using the mean value of pipette solution volume $\left(150,575 \mu \mathrm{m}^{3}\right)$, Figure 9 shows a plot of achievable CDF versus cell diameter. CDF decreases cubically as cell diameter increases and hence, with cells greater than $40 \mu \mathrm{m}$ diameter, whole-cell recordings should be possible with the CDF at 10 or lower.

Use of Mineral Oil: Mineral oil has been used previously to improve seals in giant patches [21] while obtaining cellattached configuration. To the best of our knowledge there are no reports of the solubility of cytosolic constituents in mineral oil. It seems improbable that the water soluble components of the cytosol will diffuse into the mineral oil. If vesicles or organelles migrate into the pipette solution they might interact with mineral oil, as might the plasma membrane, but we have seen no evidence of this.

Comparison with the perforated patch approach: The perforated patch approach is the current gold standard for preserving cytosolic integrity while allowing voltage and current clamp recordings from cell membranes. However, with the exception of the membrane pores generated with $\beta$-escin, pores generated chemically are small in size and prohibit the application of test compounds to the pipette solution [12]; they would be unable to enter the cytosol. As pore size is increased, larger cytosolic entities can diffuse into the pipette solution. With the approach we describe here to minimize cytosol dilution, it is possible to add compounds of any size into the pipette solution (as we demonstrated by adding FSAT). Another drawback of the perforated patch technique is that it increases series resistance over the conventional whole-cell method, something we avoid.

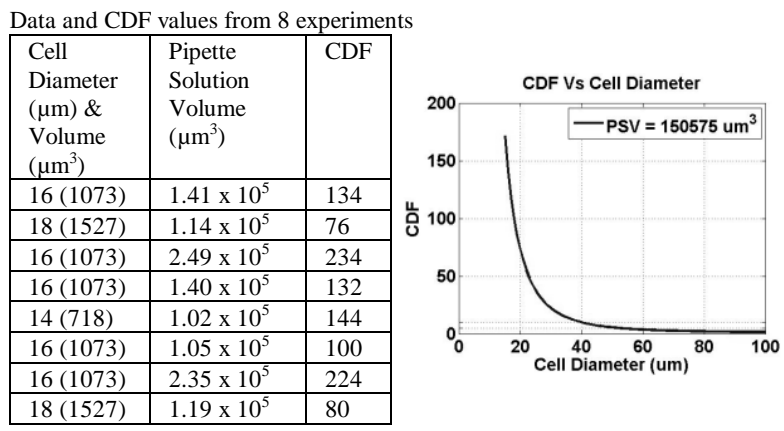

Fig. 9. Table shows the CDF values obtained in 8 experiments. Mean CDF is 140 with a standard deviation of 60 . The plot shows CDFs achievable with different cell diameters using the mean value of pipette solution volume (addional grid lines in the plot are for CDF values of 5 and 10). CDF less than ten is achievable with cells having bodies with diameters $40 \mu \mathrm{m}$ or greater.

\section{CONCLUSION}

We have introduced a novel pipette holder for whole-cell recording with the goal of minimizing cytosol dilution. To the best of our knowledge, ours is the first attempt to minimize cytosol dilution for patch clamp systems (but see [22]). Our method brings the whole-cell patch-clamp recording conditions one step closer to the ideal, where there is no cytosol dilution 
and with series resistance unaffected. We have demonstrated whole-cell configuration can be obtained with $\mathrm{CHO}$ cells obtaining cytosol dilution factors between 76 and 234. We believe that with other cell types, it would be equally feasible to achieve a low CDF whole-cell configuration. With larger cells (having diameters greater than $40 \mu \mathrm{m}$ ), dilution factors less than 10 would be achievable with no improvements to our system. With the ability to add test compounds to the pipette solution, the whole-cell recordings with this new system should be able to generate interesting data from patch-clamp experiments that will be useful in furthering our understanding of cellular physiology.

Future work includes; 1) Use of current setup and cells having larger diameter, to demonstrate prevention of signal rundown during whole-cell experiments. 2) Improvement of the novel pipette holder to permit cytosol dilution factors of less than 5 with cells having somas close to $10 \mu \mathrm{m}$ diameter. 3) Incorporation of a methodology to control the cytosol dilution factor sequentially from a lower to a higher value to study the effect of cytosol dilution on cellular physiology.

\section{ACKNOWLEDGMENT}

We thank Professors Alan Sahakian (Electrical Engineering and Computer Science Department, Northwestern University) and Joshua Singer (Ophthalmology and Physiology Department, Northwestern University) for their suggestions and comments on the work, Professor Robert Lamb (Molecular Biosciences Department, Northwestern University) for providing the $\mathrm{CHO}-\mathrm{K} 1$ cell line and Dr. Chunlong $\mathrm{Ma}$ (Neurobiology and Physiology Department, Northwestern University) for his help in the laboratory and with cell culture.

\section{REFERENCES}

1. Neher, E. and B. Sakmann, Single-channel currents recorded from membrane of denervated frog muscle fibres. Nature, 1976. 260(5554): p. 799-802.

2. Hamill, O.P., et al., Improved patch-clamp techniques for high-resolution current recording from cells and cell-free membrane patches. Pflugers Arch, 1981. 391(2): p. 85-100. 3. Inayat, S., et al., A Novel Way to Go Whole-Cell in PatchClamp Experiments. IEEE Trans Biomed Eng, 2010. 57(11): p. $2764-2770$.

4. Marty, A. and E. Neher, Chapter 7 Tight-Seal Whole-Cell Recording. Single-channel recording, ed. B. Sakmann and E. Neher1983, New York: Plenum Press. xxii, 503 p.

5. Godt, R.E. and D.W. Maughan, On the composition of the cytosol of relaxed skeletal muscle of the frog. Am J Physiol, 1988. 254(5 Pt 1): p. C591-604.

6. Reed, J.L., et al., An expanded genome-scale model of Escherichia coli K-12 (iJR904 GSM/GPR). Genome Biol, 2003. 4(9): p. R54.

7. Luby-Phelps, K., Cytoarchitecture and physical properties of cytoplasm: volume, viscosity, diffusion, intracellular surface area. Int Rev Cytol, 2000. 192: p. 189-221.
8. Marty, A. and J. Zimmerberg, Diffusion into the patchclamp recording pipette of a factor necessary for muscarinic current response. Cell Signal, 1989. 1(3): p. 259-68.

9. Fenwick, E.M., A. Marty, and E. Neher, Sodium and calcium channels in bovine chromaffin cells. J Physiol, 1982. 331: p. 599-635.

10. Belles, B., et al., "Run-down" of the Ca current during long whole-cell recordings in guinea pig heart cells: role of phosphorylation and intracellular calcium. Pflugers Arch, 1988. 411(4): p. 353-60.

11. Korn, S.J. and R. Horn, Influence of sodium-calcium exchange on calcium current rundown and the duration of calcium-dependent chloride currents in pituitary cells, studied with whole cell and perforated patch recording. J Gen Physiol, 1989. 94(5): p. 789-812.

12. Sarantopoulos, C., Perforated Patch-Clamp Techniques, 2007. p. 253-293.

13. Fenwick, E.M., A. Marty, and E. Neher, A patch-clamp study of bovine chromaffin cells and of their sensitivity to acetylcholine. J Physiol, 1982. 331: p. 577-97.

14. Sherman-Gold, R., Instrumentation for measuring bioelectric signals from cells. 2nd ed. The Axon guide for electrophysiology \& biophysics: laboratory techniques, ed. E.

R. Sherman-Gold1993: The Axon guide for electrophysiology \& biophysics: laboratory techniques, Molecular Devices. 60.

15. Horn, R. and S.J. Korn, Prevention of rundown in electrophysiological recording. Methods in Enzymology, 1992. 207(8): p. 149-155.

16. Walz, W., Perforated Patch-Clamp Technique, PatchClamp Applications and Protocols, A.A. Boulton, G.B. Baker, and W. Walz, Editors. 1995, Humana Press. p. 155-171.

17. Dufy, B., A. MacDermott, and J.L. Barker, Rundown of GH3 Cell $\mathrm{K}^{+}$conductance response to TRH following patch recording can be obviated with $\mathrm{GH} 3$ cell extract. Biochemical and Biophysical Research Communications, 1986. 137(1): p. 388-396.

18. Rosenmund, C. and G.L. Westbrook, Rundown of $N$ Methyl-D-Aspartate channels during whole-cell recording in rat hippocampal neurons: Role of $\mathrm{Ca}^{2+}$ and ATP. Journal of Physiology, 1993. 470: p. 705-729.

19. Strauss, U., et al., Whole-cell patch-clamp: true perforated or spontaneous conventional recordings? Pflugers Arch, 2001. 442(4): p. 634-8.

20. Dickmann, K., F. Demming, and J. Jersch, New etching procedure for silver scanning tunneling microscopy tips. Review of Scientific Instruments, 1996. 67(3): p. 845-846. 21. Couey, J.J., et al., Giant excised patch recordings of recombinant ion channel currents expressed in mammalian cells. Neurosci Lett, 2002. 329(1): p. 17-20.

22. Verma, P. and N.A. Melosh, Gigaohm resistance membrane seals with stealth probe electrodes. Applied Physics Letters, 2010. 97: p. 33704-1 - 33704-3. 